PROCEEDINGS OF THE

AMERICAN MATHEMATICAL SOCIETY

Volume 127, Number 8, Pages 2437-2442

S 0002-9939(99)04716-4

Article electronically published on March 24, 1999

\title{
DEHN SURGERY ON THE FIGURE 8 KNOT: IMMERSED SURFACES
}

\author{
I. R. Aitchison, S. MATSUmoto, AND J. H. RUBINSTEIN
}

(Communicated by Ronald A. Fintushel)

\begin{abstract}
It is known that about $70 \%$ of surgeries on the figure 8 knot give manifolds which contain immersed incompressible surfaces. We improve this to about $80 \%$ by giving a very simple proof that all even surgeries give manifolds containing such a surface. Moreover, we give a quick proof that every $(6 k, t)$ surgery is virtually Haken, thereby partially dealing with some exceptional cases in Baker's results.
\end{abstract}

\section{INTRODUCTION}

Perhaps the best studied knot complement is that of the figure eight knot, or Listing's knot. We will denote the complement by $M_{8}$. A brief description of $M_{8}$ can be found in Thurston [Th2], with more detail in [Th1]. The analysis in [Th1] is facilitated by the simplicity of the combinatorial structure of $M_{8}$ : it is the union of two tetrahedra, with corresponding combinatorial decomposition of its universal cover being the tessellation of hyperbolic 3-space by regular ideal hyperbolic tetrahedra. Thurston [Th1] showed that all but finitely many surgeries on the figure eight knot yield non-Haken hyperbolic 3-manifolds. Therefore no closed, incompressible surfaces exist in these manifolds.

It is conjectured that every closed hyperbolic 3-manifold

- contains an immersed incompressible surface of negative Euler characteristic,

- is finitely covered by a Haken manifold,

- has virtually Z-representable fundamental group, and

- is a virtual bundle over $S^{1}$.

These conjectures are consecutively stronger. For further references and discussion on the existence of immersed $\pi_{1}$-injective surfaces or Haken covers, we refer the reader to Problems 3.2, 3.7 and 3.50 of Kirby's recently updated problem list [Ki].

Reid [Re] has shown that the figure eight knot is the only arithmetic knot. It is thus important to understand the structure of $M_{8}$ in order to ascertain the extent to which its properties either arise from its arithmeticity, from its particularly simple combinatorial structure, or are generic.

Received by the editors November 12, 1996 and, in revised form, October 21, 1997.

1991 Mathematics Subject Classification. Primary 57Q35; Secondary 57M50.

Key words and phrases. Cubed manifolds, immersed incompressible surfaces, dihedral cover, Dehn surgery.

This research was partially supported by the Australian Research Council.

(C)1999 American Mathematical Society 
Denote by $M_{(p, q)}$ the closed orientable 3-manifold obtained from $M_{8}$ by $(p, q)$-Dehn surgery. Several authors have addressed virtual Z-representability of $\pi_{1}\left(M_{(p, q)}\right)$ (conjecture 3 above), obtaining results by producing covers with nonperipheral homology. Their combined efforts show that essentially $70 \%$ of possibilities are currently known to have virtually $\mathbf{Z}$-representable $\pi_{1}$, and thus essentially $70 \%$ contain an incompressible closed immersed surface of genus greater than 1 .

We address conjecture 1 directly, and proceed by juxtaposing several (by now) fairly well-known observations. Our main result is

Theorem 1.1. For every $k, q \neq 0, M_{(2 k, q)}$ contains an immersed incompressible surface of genus greater than or equal to 2 .

Corollary 1.2. Essentially $80 \%$ of 3-manifolds obtained by surgery on $M_{8}$ contain an incompressible closed immersed surface of genus greater than 1.

Theorem 1.3. For every $k, q \neq 0, M_{(6 k, q)}$ is virtually Haken.

This enables us to partially deal with half of the exceptional cases in one of Baker's results: in $[\mathrm{Ba}]$ it is shown that for every $k, M_{(3 k, q)}$ has virtually $\mathbf{Z}$ representable fundamental group, unless $q=k \pm 1$.

\section{Preliminaries}

The figure eight knot complement $M_{8}$ is fibred of genus 1 , is isotopic to its mirror image (amphichirality), and can be oriented in only one way up to isotopy in $S^{3}$. Hence, using any standard choice of oriented meridian $\mu$ and longitude $\lambda$ to coordinatize Dehn surgery, we may assume that any 3-manifold obtained by surgery on $M_{8}$ is determined by a pair of relatively prime integers $(p, q)$, with $p, q \geq 0$. We let $M_{(p, q)}$ denote the closed manifold obtained by gluing in a solid torus $S^{1} \times D^{2}$ to $M_{8}$ with the boundary of the disc corresponding to the simple closed curve $p \mu+q \lambda$ on a peripheral torus of $M_{8}$. Subsequently, we will assume that $p \geq 1, q \neq 0$.

A closed orientable 3-manifold is irreducible if every embedded 2-sphere is the boundary of an embedded 3-ball, and is a Haken manifold if it also contains an embedded closed surface of genus at least 1 with $\pi_{1}$-injective fundamental group. We will call such a surface an incompressible surface. An irreducible closed orientable 3-manifold without such a surface is a non-Haken 3-manifold. A manifold is virtually Haken if it has a finite-sheeted Haken cover.

Thurston [Th1] showed that all Dehn surgeries except for $(1,0),(0,1),(1,1)$, $(2,1),(3,1)$ and $(4,1)$ yield non-Haken hyperbolic 3 -manifolds.

An irreducible manifold $M$ has virtually $\mathbf{Z}$-representable fundamental group if it has a finite-sheeted cover $\tilde{M}$ with first Betti number

$$
\beta_{1}=\operatorname{rank}\left(H_{1}(\tilde{M}, \mathbf{R})\right) \geq 1 .
$$

Thus $\tilde{M}$ is Haken, and $M$ then contains an immersed, incompressible surface.

It is known that $\pi_{1}\left(M_{(p, q)}\right)$ is virtually $\mathbf{Z}$-representable in the following cases:

$$
\begin{aligned}
q & \equiv \pm 2 p(\bmod 7) \\
q & \equiv \pm p(\bmod 13) \\
p & \equiv 0(\bmod 4), \\
p & \equiv 0(\bmod 3), q \pm 1 \neq p / 3 \\
p & \equiv 0(\bmod 2), q \equiv \pm 7 p(\bmod 15)
\end{aligned}
$$

(see respectively $[\mathrm{N}]$ or $[\mathrm{He} 1] ;[\mathrm{He} 1] ;[\mathrm{Pr}],[\mathrm{KL}],[\mathrm{Mo}]$ and $[\mathrm{Ba}] ;[\mathrm{Ba}] ;[\mathrm{Ba}]$ ). 


\section{THE DIHEDRAL COVER}

Lemma $3.1([\mathrm{Bu}])$. The 5-fold irregular dihedral cover of the figure eight knot complement is homeomorphic to the complement $M_{8}^{*}$ of the link $8_{4}^{3}$ in $S^{3}$.

Remark. Fox [Fo1], [Fo2] had shown that many potential counterexamples to the Poincaré Conjecture can be constructed by taking irregular branched covers of a knot or link in $S^{3}$ : any potential counterexample arises as some branched cover. Burde $[\mathrm{Bu}]$ showed that many examples in Fox's 'bushel basket' of homotopy 3spheres are standard spheres. A concrete example is described in [Bu]: Burde showed that the 5 -fold irregular dihedral cover of the figure 8 knot complement can be realized (non-uniquely) as a link complement in $S^{3}$, and provided a specific example. His example is exactly the link $8_{4}^{3}$ (Rolfsen's notation, [Ro]): Burde actually gives a picture of this link.

Note that since each component of $8_{4}^{3}$ is unknotted, there are infinitely many distinct such links with homeomorphic complements. It is therefore serendipitous that Burde provided the most desirable possibility for our purposes.

To see why the 5 -fold irregular covering of $M_{8}$ should be a link complement, observe that the two fold branched covering of $S^{3}$, with branch set the figure 8 knot, is the lens space $L(5,2)$, which in turn is freely 5 -fold covered by $S^{3}$. The 5 -fold irregular branched dihedral cover is then obtained by taking any $\mathbf{Z}_{2}$ action on $S^{3}$ with fixed point set one of the five lifts of the branch set in the lens space; this action interchanges the remaining two pairs of components, and has quotient $S^{3}$ containing a 3 -component link - the link $8_{4}^{3}$.

The link $8_{4}^{3}$ has been discussed in [Ha], [AR] and [AMR1]. Hatcher remarked that the cubical decomposition of $M_{8}^{*}$ was compatible with the subdivision of the regular ideal cubing of $\mathbf{H}^{3}$ into the regular tetrahedral decomposition noted by Thurston [Th1]. The cubical structure had been expoited in [ALR] to show that most surgeries gave manifolds containing immersed incompressible surfaces. Hass and Menasco $[\mathrm{HM}]$ showed that infinitely many of these examples were non-Haken, and in [AMR1], we observed that infinitely many of the Hass-Menasco examples are virtually Haken. Virtual Z-representability of the fundamental groups was not addressed in these papers.

In [AMR2], face identifications of the tetrahedral subdivision of two ideal cubes were given showing the existence of a covering projection to $M_{8}$, and in seeking a concrete realization of this 5 -fold covering space we were led to Burde's paper [Bu].

The regular 10-fold dihedral cover of $M_{8}$ can now easily be drawn as a link complement $M_{8}^{* *}$ in $S^{3}$. It is also amusing to observe that the link complement $N=S^{3}-C(3,-2)$ arising in Neumann and Reid's paper [NR] has complement which is 2 -fold covered by $M_{8}^{* *}$ : however, $N$ is not a 5 -fold cover of $M_{8}$. We would like to thank Jeff Weeks and Craig Hodgson for their timely provision of the latest version of Snappea, which determines covers and symmetry groups and decides whether or not two (small) specified hyperbolic manifolds are isometric.

\section{LIFTING SURGERIES}

We will say that a surgery on $M_{8}$ lifts to a surgery on $M_{8}^{*}$ if the surgery curve lifts to closed curves on each peripheral torus of $M_{8}^{*}$, each covering the original 1:1. This is equivalent to $M_{(s, t)}$ being freely covered by the associated surgery $M_{(s, t)}^{*}$ on $M_{8}^{*}$. 
Lemma 4.1. An $(s, t)$ surgery on $M_{8}$ lifts to a surgery on $M_{8}^{*}$ if and only if $s=2 k$ for some integer $k$.

Proof. The shape of the cusp torus of $M_{8}$ is determined by its fundamental domain, which is a strip of eight equilateral triangles (one for each ideal vertex of the two tetrahedra), as illustrated in Figure 1 (see [Th1] or [ALR]); the meridian corresponds to the edges marked with an arrow, which are identified. Unwrapping twice in the meridianal direction yields a cusp with two strips of eight triangles; a $(p, q)$ curve on the peripheral torus of $M_{8}$ lifts to one or two curves, according to whether $p$ is odd or even.

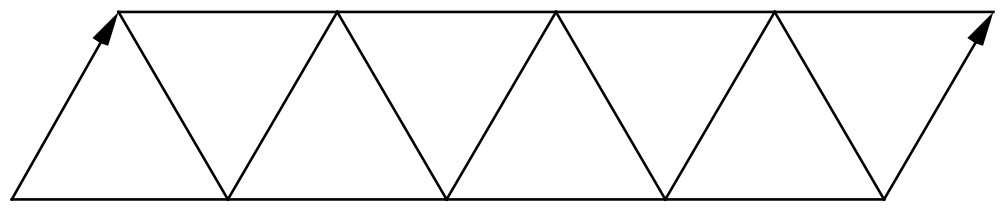

Figure 1. Figure-8 knot cusp torus fundamental domain

\section{IMMERSED SURFACES}

Theorem 5.1. If $M_{(2 k, t)}$ is hyperbolic, then it contains an immersed, closed, incompressible surface.

Proof. It is shown in [Ha] and $[\mathrm{AR}]$ that the link $8_{4}^{3}$ is a balanced link, with $M_{8}^{*}$ consisting of two regular ideal hyperbolic cubes, with all edges of degree 6 . With respect to the cubical structure, the three cusp tori have fundamental domains which consist of strips of 4,4 and 8 equilateral triangles respectively, as can be seen directly by counting the number of crossings. Lifting the cusp structure from $M_{8}$, we see that the two cusps with 4 triangles actually have each of their triangles subdivided into 4 triangles, thereby creating a double strip of 8 triangles: these are the cusps double covering the peripheral torus of $M_{8}$. (See also [Ha].)

It is shown in [ALR] that there is a canonical totally geodesic surface $S$ immersed in $M_{8}^{*}$, intersecting each of the cubes in three squares orthogonal to the edges, each of which bisect the cube. Furthermore, $S$ survives as an immersed incompressible surface after any Dehn surgery, except for at most 12 . Of the possibly excluded surgeries, the only even ones to be avoided (after accounting for symmetry of $M_{8}$ ) are $(0,1),(2,1)$ or $(4,1)$. But these are exactly those appearing in Thurston's list of exclusions to a hyperbolic structure on $M_{(p, q)}$ given earlier.

Corollary 5.2. Essentially $80 \%$ of 3-manifolds obtained by surgery on $M_{8}$ contain an incompressible closed immersed surface of genus greater than 1.

This follows by carefully estimating the proportion of manifolds now known to have this property. Previous results show that essentially $70 \%$ of surgeries give manifolds containing an incompressible closed immersed surface of genus greater than 1 . This is calculated by determining the number of solutions of the congruence equations given earlier, and accounting for duplication by use of the Chinese Remainder Theorem for solving simultaneous congruences. This is easily done due to many of the moduli being relatively prime. A similar, but easier, calculation shows that we now have about $80 \%$ of cases covered. 


\section{HAKEN COVERS}

Theorem 6.1. $M_{(6 k, t)}$ is virtually Haken.

Proof. In [AMR1] we proved that there is a 6 -fold cover $M_{8}^{c o l}$ of $M_{8}^{*}$, the 'coloured cover', which contains a non-peripheral embedded incompressible surface. Various surgeries on $M_{8}^{*}$ lift to $M_{8}^{c o l}$; these can be determined as follows:

Label the vertices of the unit-length equilateral triangulation of the Euclidean plane by labelling the vertices of one triangle with 'colours' 1, 2, 3, extending by reflection. Choose a vertex as base point. If a cusp torus has 'shape $n$ ', it has fundamental domain the parallelogram consisting of a strip of $2 n$ triangles. This parallelogram determines a $\mathbf{Z} \oplus \mathbf{Z}$ subgroup of the symmetries of the equilateral lattice. A meridian corresponds to the translation of this equilateral lattice by 1 unit parallel to one of the sides of a triangle, and identifies one long edge of the parallelogram with the other. Any closed curve on the cusp torus then corresponds to a straight line segment joining the base point to one of its images under an element of $\mathbf{Z} \oplus \mathbf{Z}$. Such a curve lifts to a surgery on $M_{8}^{\text {col }}$ if and only if the corresponding permutation of the symbols $1,2,3$ is the identity.

The meridian thus corresponds (up to relabelling of symbols) to the cycle (123). Now a longitude $\lambda$ of $M_{8}$ lifts to $M_{8}^{*}$. Let $\lambda_{1}$ and $\lambda_{2}$ denote the corresponding lifts to the cusps of $M_{8}^{*}$ covering once and twice respectively. From [Th1], we know that $\lambda$ corresponds to a segment on the equilateral lattice which is the vector sum of the long edge (length 4) of the cusp parallelogram, together with two meridians. An alternative description is that it corresponds to a line segment passing through the basepoint, transverse to the meridianal segment, and bisecting 4 triangles. The curve $\lambda_{1}$ thus corresponds to the identity permutation, as does $\lambda_{2}$. These permutations determine a homomorphism of $\mathbf{Z} \oplus \mathbf{Z}$ to the symmetric group $S_{3}$ on three symbols, and curves which lift to $M_{8}^{c o l}$ are those in the kernel of this homomorphism. Thus we have proved the following lemma:

Lemma 6.2. A lift of $a(2 k, q)$ curve on the cusp torus of $M_{8}$ to the cusps of $M_{8}^{*}$

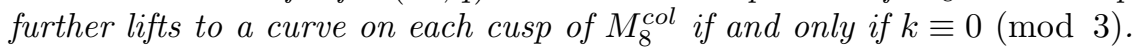

Again by [ALR], any surgery on $M_{8}$ of the form $(6 k, t)$ lifts to a surgery on $M_{8}^{*}$ which gives a manifold $M_{(6 k, t)}^{*}$ in which the canonical surface remains incompressible. In turn, such a surgery lifts to a surgery on $M_{8}^{c o l}$, with the preimage of the

canonical surface containing an embedded component. Thus $M_{(6 k, t)}$ is virtually Haken as claimed.

\section{REFERENCES}

[ALR] I.R. Aitchison, E. Lumsden and J.H. Rubinstein, Cusp structure of alternating links, Invent. Math. 109 (1992), 473-494. MR 93h:57007

[AMR1] I. R. Aitchison, S. Matsumoto, and J. H. Rubinstein, Immersed surfaces in cubed manifolds, Asian J. Math 1 (1997), 85-95. CMP 98:04

[AMR2] _ Immersed surfaces in the figure-8 knot complement, preprint, 1996.

[AR] I. R. Aitchison and J. H. Rubinstein, Combinatorial cubings, cusps and the dodecahedral knots, in: Proc. of the Special Semester on Topology at Ohio State University, 1990 (de Gruyter, Berlin - New York, 1992) pp. 17-26. MR 93i:57016

[Ba] M. D. Baker, On coverings of figure eight knot surgeries, Pacific J. Math. 150 (1991), 215-228. MR 92g:57004

[Bu] G. Burde, On branched coverings of $S^{3}$, Canad. J. Math. 23 (1971), 84-89. MR 43:6908 
[Fo1] R. H. Fox, Construction of simply connected 3-manifolds, in: Topology of 3-manifolds and related topics, Proc. The Univ. of Georgia Institute, 1961 (Prentice-Hall, Englewood Cliffs, N.J., 1962) pp. 213-216. MR 25:3539

[Fo2] R. H. Fox, Metacyclic invariants of knots and links, Canad. J. Math. 22 (1970), 193-201. MR 41:6197

[HM] J. Hass and W. Menasco, Topologically rigid non-Haken 3-manifolds, J. Austral. Math. Soc. (Series A) 55 (1993), 60-71. MR 94g:57017

[Ha] A. Hatcher, Hyperbolic structures of arithmetic type on some link complements, J. London Math. Soc. (2) 27 (1983), 345-355. MR 84m:57005

[He1] J. Hempel, Coverings of Dehn fillings of surface bundles, Topology Appl. 24 (1986), 157-170. MR 88h:57011

[Ki] R.C. Kirby (ed.), Problems in low-dimensional topology, in: Geometric Topology (2 vols), 1993 Georgia International Topology Conference Proceedings (ed. William H. Kazez) (American Mathematical Society, Providence, 1997). CMP 98:01

[KL] S. Kojima and D. Long, Virtual Betti numbers of some hyperbolic 3-manifolds, in: A fete of topology: Papers dedicated to Itiro Tamura (Academic Press, New York, 1988), pp.417-437. MR 89a:57060

[Mo] S. Morita, Finite coverings of punctured torus bundles and the first Betti number, Sci. Papers College Arts Sci. Univ. Tokyo 35 (1986), no. 2, 109-121. MR 88b:57021

[NR] W. D. Neumann and A. W. Reid, Arithmetic of hyperbolic manifolds, in: Proc. of the Special Semester on Topology at Ohio State University, 1990 (de Gruyter, Berlin - New York, 1992) pp. 273 - 310. MR 94c:57024

[N] A. Nicas, An infinite family of non-Haken hyperbolic 3-manifolds with vanishing Whitehead groups, Math. Proc. Camb. Phil. Soc. 99 (1986), 239-246. MR 87h:57018

[Pr] J. H. Przytycki, Incompressibility of surfaces after Dehn surgery, Michigan Math. J. 30 (1983), 289-308. MR 86g:57012

[Re] A. W. Reid, Arithmeticity of knot complements, J. London Math. Soc. (2) 43 (1991), 171-184. MR 92a:57011

[Ro] D. Rolfsen, Knots and Links (Publish or Perish, Inc., Houston, 1990). MR 95c:57018

[Th1] W.P. Thurston, The geometry and topology of 3-manifolds, Princeton University Lecture Notes, 1978.

[Th2] W.P. Thurston, Three-dimensional Geometry and Topology, Princeton Mathematical Series \#35 (Princeton University Press, Princeton, 1997). MR 97m:57016

Department of Mathematics, University of Melbourne, Parkville, Victoria 3052 , Australia

E-mail address: iain@maths.mu.oz.au

E-mail address: saburo@is.titech.ac.jp

E-mail address: rubin@maths.mu.oz.au 